\title{
DRUG DELIVERY TO THE BRAIN USING POLYMERIC NANOPARTICLES: A REVIEW
}

\author{
Bhatt Neha*, Bhatt Ganesh and Kothiyal Preeti \\ Shri Guru Ram Rai Institute of Technology \& Sciences Dehradun, Uttarakhand, India
}

\begin{abstract}
Nanoparticle drug carriers consist of solid biodegradable particles in size ranging from 10 to $1000 \mathrm{~nm}$ (50-300 nm generally). The use of minute particles as drug carriers for targeted treatment has been studied over a long period of time. A selective accumulation of active substances in target tissues has been demonstrated for certain so-called nanocarrier systems that are administered bound to pharmaceutical drugs. Great expectations are placed on nanocarrier systems that can overcome natural barriers such as the blood-brain barrier (BBB) and transport the medication directly to the desired tissue and thus heal neurological diseases that were formerly incurable. Polymeric Nanoparticle have been shown to be promising carriers for CNS drug delivery due to their potential both in encapsulating drugs, hence protecting them from excretion and metabolism, and in delivering active agents across the blood - brain barrier without inflicting any damage to the barrier. Different polymers have been used and different strategies like surface modification have been done to increase the retention time of nanoparticles.
\end{abstract}

Key words: Nanoparticle, blood-brain barrier, surface modification, reticuloendothelial system

\section{Introduction}

The global market for drugs for the central nervous system (CNS) is greatly underpenetrated and would have to grow by over $500 \%$ just to be comparable to the global market for cardiovascular drugs ${ }^{[1]}$.

The main reason is that why neurotherapeutics are unsuccessful in treating CNS disorders because they cannot be effectively delivered the required concentration of drug in the brain because of relatively impermeable Blood Brain Barrier and as a result several potential molecules are lost from the market. ${ }^{[2]}$

Correspondence to author:

Neha Bhatt (M.Pharma, Pharmaceutics)

Shri Guru Ram Rai institute of Technology \& Sciences

Dehradun, Uttarakhand,

Mobile no - 09760371229

E-mail: nehabhatt.ddn@gmail.com 
It is now evident that neurologic disorders have emerged as priority health problems worldwide. This is reflected in the Global Burden of Disease Study, jointly published by the World Health Organization and other groups. The proportionate share of the total global burden of disease resulting from neuropsychiatric disorders is projected to rise to $14.7 \%$ by 2020 . Although neurologic and psychiatric disorders comprise only $1.4 \%$ of all deaths, they account for a remarkable $28 \%$ of all years of life lived with a disability. ${ }^{[3]}$

The World Health Organization (WHO) estimates that 700 million cases of motor neuron diseases (MND) are reported annually-accounting for $13 \%$ of global disease burden. Out of this number, 150 million live with depression, 25 million live with schizophrenia, 38 million live with epilepsy, and 90 million live with a substance abuse disorder. A number of factors are responsible for increasing the disease burden: insufficient focus on basic mental health care, lack of funding of prevention programs, and the stigma associated with these disorders. Estimates show this disease area will become the leading cause of disability in two decades. ${ }^{[4]}$ Annually, mental disorders lead to 1 million suicides worldwide ${ }^{[5]}$.

One mental condition, major depressive disorder, is projected to be the worldwide leading cause of disease burden by $2030^{[6]}$ Alzheimer's disease, a neurological disorder for which there is not yet a cure, affected 36 million people globally in 2010 and is expected to rise steeply to 115 million by $2050^{[7]}$

Thus there are various approaches attempting to reach efficient concentrations of the drug in the brain include high-dose therapy or invasive methods (such as intraventricular drug infusion or temporary BBB disruption in the case of brain tumors).but, in both cases, the therapeutic effect is achieved at the expense of deleterious side effects. So far, the non-invasive systemic delivery of the drugs to the brain remains a challenge that gives rise to the development of new drug-targeting technologies. ${ }^{[8]}$

The non-invasive systemic delivery of the drugs to the brain always remains a challenge that gives rise to the development of new drug-targeting technologies. ${ }^{[9]}$

Therefore the field of novel drug delievery has been emerged into existence as an ideal approach of brain drug targeting to brain. It mainly include of use colloidal particles. The basic reason of this carrier acceptance is due to controlled profile or drug release nature as well as due to their selected targeting mechanism. ${ }^{[10]}$

In general, colloidal drug carriers include micelles, emulsions, liposomes and nanoparticles (nanospheres and nanocapsules). It is noteworthy that only liposomes and nanoparticles have been largely exploited for brain drug delivery. The aim in using colloidal carriers is generally to increase the specificity towards cells or tissues, to improve the bioavailability of drugs by increasing their diffusion through biological membranes and/or to protect them against enzyme inactivation. Moreover, the colloidal systems allow access across the BBB of non-transportable drugs by masking their physico-chemical characteristics through their encapsulation in these systems. ${ }^{[11]}$ 
Liposomes $^{[12]}$ have been used as potential drug carriers instead of conventional dosage forms because of their unique advantages which include ability to protect drugs from degradation, target the drug to the site of action and reduce the toxicity or side effects but there are some problems that have been faced like liposomes has been limited due to inherent problems such as low encapsulation efficiency, rapid leakage of water-soluble drug in the presence of blood components and poor storage stability. On the other hand, polymeric help to increase the stability of drugs proteins and possess useful Controlled release properties. ${ }^{[13]}$ the low number of excipients used in their formulations, the simple procedures for preparation, a high physical stability, and the possibility of sustained drug release that may be suitable in the treatment of chronic diseases ${ }^{[14]}$ so the non-invasive systemic drug delivery to the brain by means of the nanoparticulate carriers and it appears to be a promising option.

Nanoparticles are solid colloidal particles, ranging in size from 1 to $1000 \mathrm{~nm}$ (usually 200-300 $\mathrm{nm}$ ), and made of polymers or lipids, "nanosphere" is used to identify a nanoparticle system with a matrix character and constituted by a solid core with a dense polymeric network. In contrast, "nanocapsules" are formed by a thin polymeric envelope surrounding an oil-filled cavity. Nanocapsules may, thus, be considered as a "reservoir" system.

Practically, the nanoparticles have a size around $200 \mathrm{~nm}$ and the drugs or other molecules may be dissolved into the nanoparticaes, entrapped, encapsulated and/or adsorbed or attached. ${ }^{\text {[15] }}$ Here, we review we discuss the role of blood brain barrier as a obstacle in delivery of drugs role of polymeric nanoprticles as drug delivery device for brain targeting, their method of preparation, modification of surface properties to increase the retention time of nanoparticles and drug release.

\section{Blood-brain barrier (BBB) an major obstacle of drug delivery to CNS}

The blood - brain barrier (BBB) is a diffusion barrier, which impedes influx of most compounds from blood to brain while supplying the brain with the required nutrients for proper function.

Unlike peripheral capillaries that allow relatively free exchange of substances across/between cells, the BBB strictly limits transport into the brain through both physical (tight junctions) and metabolic (enzymes) barriers . ${ }^{[16]}$ Thus the BBB is often the rate-limiting factor in determining permeation of therapeutic drugs into the brain. (Figure 1)

The structural difference between the brain capillaries and the non brain capillary endothelium is associated with the endothelial tight junctions.

The non brain capillaries have fenestrations (openings) between the endothelial cells through which solutes can move readily via passive diffusion, whereas in brain capillaries, the endothelium epithelial is unfenestrated (tight junctions without openings). A low amount of pinocytosis vesicles and particular tight junctions (TJs) also known as zonula occludens which Divide the membranes of the endothelial cells into two distinct sides, luminal (blood side) and abluminal (brain side). 
Thus the presence of tight junctions and absence of pinocytosis thus eliminates a paracellular across brain capillary endothelium pathway of solute movement across the BBB [17]. TJs are structures that form a narrow and continuous seal surrounding each endothelial and epithelial cell at the apical border and are aimed at strictly regulating the movement of molecules through the paracellular pathway ${ }^{[18] .}$

The BBB endothelial TJs show some differences in morphology, composition, and complexity features from those of both the epithelia and peripheral endothelia ${ }^{[19]}$. These unfenestrated capillaries which are cemented together by intercellular tight junctions their electrical resistance across intraparenchymal endothelial cells may be as high as $2000 \Omega \mathrm{cm} 2$. ${ }^{[20]}$ A further contribution to the peculiar BBB functions is given by the periendothelial accessory structures represented by astrocytes ${ }^{[21,22]}$, pericytes ${ }^{[24,23,25]}$, and the basal membrane.

Astrocytic end-feet processes form around $99 \%$ of the CNS capillaries, yet they do not contribute to the physiological activity of the BBB. They however secrete atropic factor necessary for the differentiation, health and function of the BBB endothelial cells.

Astrocytes, generally classified into fibrous and protoplasmic, represent the major component ( $90 \%)$ of the brain mass.

Fibrous astrocytes have a star-like morphology and often present many long processes, known as "end-feet", that end on the basal membrane of the BBB). These cells have a multitude of functions important for the brain homeostasis (maintenance of $\mathrm{K}+$ levels, inactivation of neurotransmitters, regulation and production of growth factors and cytokines), many of which are related to the production of apolipoprotein $E(A p o E)$

Attached at irregular intervals to the abluminal membrane of the endothelium are pericytes. Pericytes and endothelial cells are unsheathed by the basal lamina.

Pericytes are spherical cells holding a prominent nucleus and many primary and secondary lysosomes in the cytoplasm and seem to be virtually involved in the BBB formation, differentiation processes, and integrity

The basal lamina is contiguous with the plasma membranes of astrocyte end-feet, which unsheathe cerebral capillaries ${ }^{[26]}$. The interrelationship between the endothelium, the pericyte and the astrocyte foot processes is as intimate as any cell-cell interactions in biology. The space filled by the basement membrane and situated between the endothelium/pericyte and the astrocyte foot process forms the interface between blood and brain.

Generally it was considered that a molecule has to possess peculiar physicochemical characteristics to overcome the BBB, such as high lipophilicity and a molecular weight less than 500 Dalton ${ }^{[27]}$ but the presence of the transport systems (generally divided in 3 classes, the presence of BBB transport systems further complicates this scenario these transporters may assist or hinder the delivery of drugs to the brain. 
The transport system has been divided into three major groups: carrier-mediated transport, active efflux transport, and receptor-mediated transport. All of them do not operate equally well in both the blood-to-brain and brain-to-blood direction.

The carrier-mediated transport (e.g. transporter proteins for glucose, essential amino acids, monocarboxylic acids, and nucleosides) ${ }^{[28,29] .}$

The use of the receptor-mediated transport to gain access to the brain is another very attractive strategy. Receptors for endogenous large molecules (e.g. insulin, transferrin, leptin , Apo, and many others) highly expressed on the BBB luminal side, are responsible for their shuttling to the brain by transcytosis. The binding of drugs or drug containers (e.g. liposomes and NPs) to specific ligands (peptides) or peptidomimetic monoclonal antibodies makes it possible to ship the construct into the brain by mimicking an endogenous molecule ${ }^{[30,31]}$.

Unfortunately, the presence of active efflux transporters to the BBB ${ }^{[32]}$ also limits the therapeutic efficacy of drugs virtually able to access the brain.e.g. P-glycoproteins; P-gp and multi drug resistance associated protein family; MRP) both on the luminal and abluminal membranes of the capillaries that regulate the transcellular traffic of essential molecules between brain and blood, as well as effluxing potentially harmful substances and waste products.

Some of the surfactants generally employed in pharmaceutical formulations have shown to inhibit P-gp and some of them are currently undergoing clinical screening for the treatment of multidrug resistant tumors. ${ }^{[33,34]}$

\section{Polymeric nanoparticle as a drug delivery tool for brain targeting}

Over the last decade polymeric nanoparticles have attracted researchers in targeting drug molecules to brain. Polymeric Nanoparticles are nanosized carriers ( $1-1000 \mathrm{~nm})$, made of natural or synthetic polymers, in which the drug can be loaded in the solid state or in solution, or adsorbed or chemically linked to the surface. Nowadays, the use of polymeric Nanoparticle is one of the most promising approaches for CNS drug delivery [35]. As name only suggest polymeric nanoparticles are nanoparticles which are prepared from polymers.

The drug is dissolved, entrapped, encapsulated or attached to a nanoparticles and depending upon the method of preparation, nanoparticles, nanospheres or nanocapsules can be obtained. Nanocapsules are vesicular systems in which the drug is confined to a cavity surrounded by a polymer membrane, while nanospheres are matrix systems in which the drug is physically and uniformly dispersed. In recent years, biodegradable polymeric nanoparticles have attracted considerable attention as potential drug delivery devices in view of their applications in drug targeting to particular organs/tissues, as carriers of DNA in gene therapy, and in their ability to deliver proteins, peptides and genes through a per oral route of administration. In spite of development of various synthetic and semi synthetic polymers, natural polymers like Chitosan, Gelatin. Sodium alginates still enjoy their popularity in drug. 
However, in recent years additional polymers are designed primarily for medical applications and have entered the arena of controlled release of bioactive agents. Many of these materials are designed to degrade within the body, most popular one are; Polylactides (PLA), Polyglycolides (PGA), Poly(lactide-co-glycolides) (PLGA), Polyanhydrides. Polyorthoesters., Polycyanoacrylates, Polycaprolactone

Originally, polylactides and polyglycolides were used as absorbable suture material. The main advantage of these degradable polymers is that they are broken down into biologically acceptable molecules that are metabolized and removed from the body via normal metabolic pathways.

However, biodegradable materials do produce degradation by-products that must be tolerated with little or no adverse reactions within the biological environment.

\section{Advantages of polymeric nanoparticles}

- Increases the stability of any volatile pharmaceutical agents, easily and cheaply fabricated in large quantities by a multitude of methods.

- They offer a significant improvement over traditional oral and intravenous methods of administration in terms of efficiency and effectiveness.

- Delivers a higher concentration of pharmaceutical agent to a desired location.

- The choice of polymer and the ability to modify drug release from polymeric nanoparticles have made them ideal candidates for cancer therapy, delivery of vaccines, contraceptives and delivery of targeted antibiotics.

- Polymeric nanoparticles can be easily incorporated into other activities related to drug delivery, such as tissue engineering

Chen et al. have aptly discussed polymeric nanoparticles as suitable delivery systems for brain

[38]. They have outlined various mechanisms for nanoparticle mediated drug uptake by the brain.

These include:

1. Enhanced retention in the brain-blood capillaries, with an adsorption on to the capillary walls, resulting in a high concentration gradient across the BBB.

2. Opening of tight junctions due to the presence of nanoparticles.

3. Transcytosis of nanoparticles through the endothelium.

But unfortunately a major disadvantage has to be by nanoparticles. After intraveneous injection, nanoparticles are taken up very rapidly by the reticuloendothelial system (RES) and distribute especially into the liver and spleen and, to a minor degree, into the bone marrow.

The RES consists of phagocytic cells originating from the bone marrow. The cells exist in the whole body, but their highest concentration is found in the liver (Kupffer cells), spleen, and bone marrow. The interaction of the colloidal carriers with blood plasma proteins (opsonins Opsonins, including complement proteins, apolipoproteins, fibronectin, and Igs,) and thus with the membranes of macrophages (opsonization) is believed to be the major criteria for clearance of these systems from the blood stream and Uptake of nanoparticles by RES could result in 
therapeutic failure due to insufficient pharmacological drug concentration build up in the plasma and hence at the BBB. ${ }^{[39,40] \text {. }}$

The extent of this uptake depends mainly on particle size, surface charge, and surface properties. A small diameter of the Np corresponds to a large relative surface area, which could promote their aggregation. Considering the surface properties, Np with a hydrophobic surface and negative charges promote protein adsorption and activate the complement system ${ }^{[41]}$. The, $\mathrm{Np}<100 \mathrm{~nm}$ in size have a lower possibility of being uptaken by macrophages or recognised by opsonins But a small diameter of the Np corresponds to a large relative surface area, which could promote their aggregation. The final size of the $\mathrm{Np}$ is an important parameter that partially determines their biological fate when administered. Particles $<10 \mathrm{~nm}$ are rapidly removed after an extensive extravasation and renal clearance, whereas $\mathrm{Np}$ of $>200 \mathrm{~nm}$ are rapidly filtrated by the spleen and removed by the reticuloendothelial cells. In addition, carriers with diameters $>5$ $\mu \mathrm{m}$ will induce capillary blockade. If the particles are physically retained in the target tissue capillaries, drug diffusion through the capillary wall (a step limited by the MW of the drug) will induce the therapeutic action. If this action requires extravasation, sizes between 0.5 and $5 \mu \mathrm{m}$ may also be suitable for that process. ${ }^{[42]}$ Like any colloidal drug carrier not especially designed to escape from MPS uptake, PLA or PLGA nanoparticles are rapidly removed from the blood stream after vascular administration and preferentially accumulate in liver and spleen.

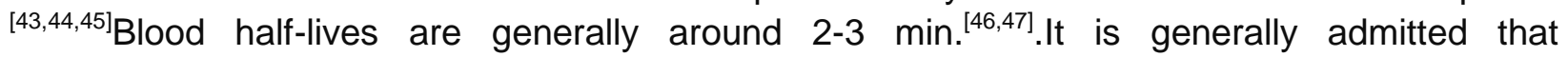
hydrophobic surfaces promote protein adsorption and that negative surfaces are activators of the complement system.

To overcome these limitations various researchers have tried to increase the plasma half-life of polymeric nanoparticles by following methods.

\section{Methods to prolong brain retention of polymeric nanoparticles}

- PEG approach is based on the long-circulating characteristics of $\mathrm{Np}$ coated or linked with PEGs.

PEG-coated NPs have received a lot of attention as they have been accounted as potentially useful tool to deliver drugs into the brain.In this respect, as early as in the '80s, different approaches were proposed for the modification of the hydrophobic particle surface; most of them were based on the physical adsorption of PEG-containing surfactants. PEG has high hydrophilicity, chain flexibility, electrical neutrality and lack of functional groups, preventing it from interacting unnecessarily with the biological components. It has been suggested that the PEG's with a molecular weight between 2000 to 5000 are necessary to suppress plasma protein adsorption; further it has been observed that the thicker the coat, the slower the clearance, and hence a better protection against liver uptake ${ }^{[48,49]}$.

More recently, surface covalent modification with PEG derivatives has been explored as an alternative strategy .All authors showed that this coating reduced NP liver uptake increasing simultaneously their persistence in the peripheral circulation as well as their deposition into other organs, including non-RES organs, for several hours. 
As previously discussed hydrophobic surfaces promote protein adsorption and that negative surfaces activate the complement system and coagulation factors so if the shielding of hydrophobic character of the nanoparticles is done then it is going to stearically stabilize them by providing a dense conformational cloud and thus reducing opsonization and phagocytosisas well as uptake by neutrophilic granulocytes, thus increasing the blood circulation time and hence the bioavailability ${ }^{[50]}$.

PEG Coating also causes Enlarging of the molecule/particle and slows down kidney ultrafiltration and, thereby allowing better accumulation into the brain and other permeable tissues by the passive enhanced permeation and retention mechanism.

It also provides protein shielding which reduces proteolysis within the serum and tissues, and hinders immune surveillance of surface epitopes. Pegylation improves the pharmacokinetic profile of molecules by reducing opsonization, phagocytosis and clearance by the liver and reticulaoendothelial system.

The conjugation of PLGA/PLA with PEG led to the development of block copolymers in which the covalent linkage of the hydrophilic PEG shell to the core of the Np avoids the possibility of PEG removal/desorption once in contact with biological fluids ${ }^{[51]}$ Other hydrophilic molecules which have been tried are Brij 78, Poloxamer F68 and Brij 68. Cavalli et al. found that parenteral administration of nanoparticles of paclitaxel was more bioavailable than an i.v. injection of the plain drug ${ }^{[52]}$.

Recently, Koziara and colleagues developed a new kind of $\mathrm{Np}$ for the BBB crossing, using emulsifying wax, Brij 72 (B-72), [53]The authors also formulated an antitumoral agent (paclitaxel), not capable of crossing the BBB, in the emulsifying wax/B-72 Np, confirming, after both in vivo and in vitro experiments, the usefulness of these Np for CNS targeting ${ }^{[53]}$

- Surfactant-based approach is based on the use of surfactants (T-80 etc.), as a coating or linked to the $\mathrm{Np}$ and acting as a target or molecule for the BBB.It was reported that nanoparticles with polysorbate 80 (Tween 80, T-80) coating represented tools used for delivering drugs to brain.Schroeder et al. ${ }^{[54]}$ studied the Radiolabeled poly(butylcyanoacrilate) (PBCA) Np coatedwith a surfactant such as T-80 and demonstrated their ability to cross the BBB when administered intravenously. Dalargin associated with PBCA NPs and polysorbate-80 induced a potent and prolonged analgesia, which was not observed by using polystyrene NPs, but not using the PBCA NPs. Locomotor activity dramatically decreased in the mice dosed with PBCA NPs, but not with the polystyrene NPs The in-vitro and in-vivo results suggested that the PBCA NPs induce a nonspecific opening of the BBB in the presence of polysorbate- 80 allowing the transport of dalargin into the CNS.

Similarly Borchardt et al. ${ }^{[55]}$ Gulyaev et al. ${ }^{[56]}$ Alyautdin et al. ${ }^{[57,58]}$ studied the polsorbate 80 coated nanoparticles and were tested and the pharmacological effects were considered as proofs of the BBB crossing. Similar results were obtained in the presence of PEGylated PBCA $\mathrm{Np}[95]$ 
Lück et al. ${ }^{[59]}$ found that apolipoprotein E (apo E) adsorbs on the surface of polysorbate 20-,40-, 60-, or 80-coated nanoparticles after $5 \mathrm{~min}$ in human plasma at 37 ' $\mathrm{C}$. After separation of the particles from the serum by centrifugation, the adsorbed plasma proteins were desorbed and analyzed by two-dimensional gel electrophoresis. For polysorbate20-,40-,6 0-, or 80-coated nanoparticles, apo $\mathrm{E}$ was found in the serum.

In contrast, without coating or with overcoating of nanoparticles using poloxamers 338 and 407, Cremophor EL, or Cremophor RH40, no adsorption of apo E was observed. The chemical nature of the overcoating surfactant is of importance, as only polysorbate-coated particles were found to show results in CNS pharmacological effect while a coating with poloxamers (184, 188, 388, or 407), poloxamine 908, Cremophors (EZ or $\mathrm{RH} 40$ ) or polyoxyethylene(23)laurylether was not effective ${ }^{[60]}$.

These results and also the studies of Lück et. al. ${ }^{[59]}$ indicate that after i.v. injection apo $E$ and/or $B$ is anchored by the polysorbate on the surface of the nanoparticles. Apo $B$ and $E$ are known to bind to lipoprotein receptors on the surface of cells. Low-density lipoprotein receptors have been identified in rat and monkey brains. They also exist in the brain capillary endothelial cells ${ }^{[61]}$. Polysorbate 80-coated nanoparticles adsorb the apolipoproteins after i.v. injection and thus seem to mimic lipoprotein particles that are able to interact with members of the low-density lipoprotein receptor family and are taken up via receptor-mediated endorytosis.B ound drugs may be further transported into the brain by diffusion. ${ }^{[62]}$

Another remarkable consideration, which may hinder the development of PBCA Np, is directly connected to the lack of FDA approval both for this polymer and for the surfactant (T-80) required for a good brain targeting result ${ }^{[63,64]}$

- Ligand-based approach is related to conjugation with specific ligands (i.e., antibodies, proteins, peptides etc.) able to increase or promote movement across the BBB crossing.

The ligand-based approach has become an interesting choice for more specific and selective drug delivery to the CNS district.This approach is based on the covalent linkage of ligands to the polymers or to the $\mathrm{Np}$ in order to promote direct interaction with transport systems ${ }^{[65,66]}$. The ligands, which could be used in CNS drug delivery, have to be chosen with appropriate characteristics in order to take advantage of receptor-mediated trancytosis or receptor-mediated endocytosis. Possible ligands could be natural substrates, such as Transferrin and OX-26 antibody, Insulin, thiamine and Peptide-derived nanoparticles but also synthetic or natural peptides

\section{Preparation of nanoparticles}

NPs have been prepared mainly by two methods:

(i) dispersion of the preformed polymers; and

(ii) polymerization of monomers

Dispersion of preformed polymers Several methods have been suggested to prepare prepare biodegradable NPs from PLA, PLG, PLGA and poly(e-caprolactone) by dispersing the 
preformed polymers. Dispersion of drug in preformed polymers is a common technique used to prepare biodegradable nanoparticles from poly (lactic acid) (PLA), poly (D, L-glycolide) (PLG), poly (D, L-lactide-co-glycolide) (PLGA) and poly (cyanoacrylate) (PCA). These can be accomplished by different methods described below.

a) Solvent evaporation

b) Nanoprecipitation

c) Emulsification/solvent diffusion

d) Salting out

e) Supercritical fluid technology (SCF)

\section{Solvent evaporation}

This technique allows the encapsulation of hydrophobic drugs firstly the polymer is dissolved in an organic solvent like dichloromethane, chloroform or ethyl acetate.

The drug is dissolved or dispersed into the preformed polymer solution, and this mixture is then emulsified into an aqueous solution to make an then emulsified into an aqueous solution to make an oil (O) in water (W) i.e., O/W emulsion by using a surfactant / emulsifying agent like gelatin, poly(vinyl surfactant / emulsifying agent like gelatin, poly(vinyl alcohol), polysorbate-80, poloxamer-188, etc

The nanosized droplets are induced by sonication or homogenization. The solvent is then

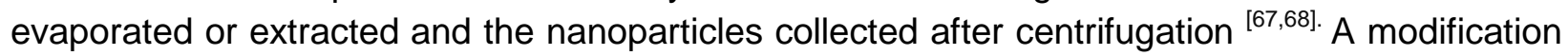
of this technique, the double emulsion $\mathrm{W} / \mathrm{O} \mathrm{W}$, was used to encapsulate hydrophilic drugs, such as peptides, proteins and nucleic acids. This method also requires high-speed homogenization or sonication. (Figure 2)

\section{Solvent displacement method and interfacial deposition}

Solvent displacement and interfacial deposition are similar methods based on spontaneous emulsification of the organic internal phase containing the dissolved polymer into the aqueous external phase (see Figure 3). However, solvent displacement forms nanospheres or nanocapsules, whereas interfacial deposition forms only nanocapsules.Solvent displacement involves the precipitation of a preformed polymer from an organic solution and the diffusion of the organic solvent in the aqueous medium in the presence or absence of a surfactant ${ }^{[70-71] .}$. The polymer, generally PLA, is dissolved in a water-miscible solvent ofi ntermediate polarity, leading to the precipitation of nanospheres.

This phase is injected into a stirred aqueous solution containing a stabilizer as a surfactant. Polymer deposition on the interface between the water and the organic solvent, caused by fast diffusion of the solvent, leads to the instantaneous formation of a colloidal suspension ${ }^{\text {[72] }}$. To facilitate the formation of colloidal polymer particles during the first step of the procedure, phase separation is performed with a totally miscible solvent that is also a non solvent of the polymer $[73,16]$. 
Considering the oil-based central cavities of the nanocapsules, high loading efficiencies are generally reported for lipophilic drugs when nanocapsules are prepared ${ }^{\text {[2]. }}$ This method is basically applicable to lipophilic drugs, and it is not an efficient means to encapsulate watersoluble drugs.. This method has been applied to various polymeric materials such as PLAn ${ }^{[74]}$, PLGA ${ }^{[75]}, \mathrm{PCL}^{[76]}$ and poly(methyl vinyl ether-comaleic anhydride) (PVM/MA) ${ }^{[77,78] .}$

Interfacial deposition is a process used for the production of nanocapsules; however, this is not a polymerization technique but an emulsification/solidification technique. In interfacial deposition, a fifth compound is introduced, of oil nature, miscible with the solvent of the polymer but immiscible with the mixture. The polymer deposits on the interface between the finely dispersed oil droplets and the aqueous phase, forming nanocapsules. An aqueous solution is used as the dispersing medium. The main difference is that polymers such as PLA are dissolved together with the drug in a solvent mixture (e.g., benzyl benzoate, acetone, and phospholipids) ${ }^{[79]}$.

This mixture is injected slowly into a stirred aqueous medium, resulting in the deposition of the polymer in the form of nanoparticles of about $230 \mathrm{~nm}$ in size. Polymer deposition occurs at the interface between water and benzoyl nanodroplets, forming nanocapsules with a shell-like wall ${ }^{[80]}$. (Figure 3)

\section{Emulsification/solvent diffusion (esd)}

In a modified version of the solvent evaporation method ${ }^{[81-83]}$ to produce the precipitation of the polymer and the consequent formation of nanoparticles, it is necessary to promote the diffusion of the solvent of the dispersed phase by dilution with an excess of water when the organic solvent is partly miscible with water or with another organic solvent in the opposite case. Subsequently, the polymer-water saturated solvent phase is emulsified in an aqueous solution containing stabilizer, leading to solvent diffusion to the external phase and the formation of nanospheres or nanocapsules, according to the oil-to-polymer ratio.

Due to the spontaneous diffusion of water-soluble solvent (acetone or methanol), an interfacial turbulence is created between two phases leading to the formation of smaller particles As the concentration of water-soluble solvent (acetone) increases, a considerable decrease in particle size can be achieved. Finally, the solvent is eliminated by evaporation or filtration, according to its boiling point. This technique presents several advantages, such as high encapsulation efficiencies (generally $>70 \%$ ), no need for homogenization, high batch-to-batch reproducibility, ease of scale-up, simplicity, and narrow size distribution.

Disadvantages are the high volumes of water to be eliminated from the suspension and the leakage of water-soluble drug into the saturated-aqueous external phase during emulsification, reducing encapsulation efficiency ${ }^{[84]}$ As with some of the other techniques, this one is efficient in encapsulating lipophilic drugs. (Figure 4) 


\section{Salting out}

Salting out with synthetic polymers: Salting -out is based on the separation of a water miscible solvent from aqueous solution via a salting-out effect.The salting-out procedure can be considered as a modification of the emulsification/solvent diffusion.

Polymer and drug are initially dissolved in a solvent such as acetone, which is subsequently emulsified into an aqueous gel containing the salting-out agent (electrolytes, such as magnesium chloride, calcium chloride, and magnesium acetate, or non- electrolytes such as sucrose) and a colloidal stabilizer such as polyvinylpyrrolidone or hydroxyethylcellulose.

This oil/water emulsion is diluted with a sufficient volume of water or aqueous solution to enhance the diffusion of acetone into the aqueous phase, thus inducing the formation of nanospheres. The selection of the salting out agent is important, because it can play an important role in the encapsulation efficiency of the drug. Both the solvent and the salting-out agent are then eliminated by cross-flow filtration. This technique used in the preparation of PLA, poly- (methacrylic) acid, and EC nanospheres leads to high efficiency and is easily scaled up. ${ }^{[85]}$

The main advantage of salting out is that it minimizes stress to protein encapsulants ${ }^{[86]}$.

Salting out does not require an increase of temperature and, therefore, may be useful when heat sensitive substances have to be processed ${ }^{[87]}$. The greatest disadvantages are exclusive application to lipophilic drugs and the extensive nanoparticle washing steps. (figure 5)

\section{Supercritical fluid technology}

Supercritical fluids is a a environmental friendly solvents, with the potential to produce PNPs with high purity and without any trace of organic solvent. ${ }^{[8]}$

The technologies are expected to offer an interesting and effective technique of particle production, avoiding most of the drawbacks of the traditional methods. Two principles have been developed for the production of nanoparticles using supercritical fluids:

1. Rapid expansion of supercritical solution (RESS)

2. Rapid expansion of supercritical solution into liquid solvent (RESOLS)

\section{Rapid expansion of supercritical solution}

In the rapid expansion of supercritical solution (RESS) method the solute of interest is solubilized in a supercritical fluid, followed by the rapid expansion of the solution across an orifice or a capillary nozzle into ambient air and the solution is expanded through a nozzle. The high degree of super saturation, accompanied by the rapid pressure reduction in the expansion, results in homogenous nucleation and thereby, the formation of well-dispersed particles. Thus, the solvent power of super- critical fluid dramatically decreases and the solute eventually precipitates.

This technique is clean because the precipitated solute is completely solvent free. 
Unfortunately, most polymers exhibit little or no solubility in supercritical fluids, thus making the technique less of practical interest. RESS was very popular in the late 80 s and early 90 s for particle production of bioerodible drug-loaded polymers like PLA. A uniform distribution of drug inside the polymer matrix can be achieved by this method for low molecular mass $(, 10000)$ polymers. However, the RESS method cannot be used for high molecular mass polymers due to their limited solubility in supercritical fluids. For these reasons, much less information is found in the literature over the past $6-7$ years on this technique..$^{89,90,91]}$

\section{Supercritical anti-solvent (SAS) method}

In the supercritical anti-solvent (SAS) method fluid in the precipitation vessel containing solute of interest in an organic solvent. At high pressures, enough anti-solvent will enter into the liquid phase so that the solvent power will be lowered and the solute precipitates. After precipitation, when the final operating pressure is reached, the anti-solvent flows through the vessel so as to strip the residual solvent. When the solvent content has been reduced to the desired level, the vessel is depressurized and the solid product is collected. In a modified version of the SAS technique ${ }^{[92],}$ the solid of interest is first dissolved in a suitable solvent and then this solution is rapidly introduced into the supercritical fluid through a narrow nozzle. The supercritical fluid completely extracts the solvent, causing the super critical fluid insoluble solid to precipitate as fine particles. This method, also called as gas anti-solvent (GAS) technique, has been successfully used to produce microparticles as well as NPs.

\section{NPs prepared from hydrophilic polymers}

Polymeric nanoparticles are prepared by using biodegradable hydrophilic polymers such as chitosan, albumin, gelatin and sodium alginate. Calvo and co-workers developed a method for preparing hydrophilic chitosan nanoparticles by ionic gelation ${ }^{[93,94]}$. Amir Dustgani et al [95] prepared Dexamethasone Sodium Phosphate loaded chitosan nanoparticles by ionic gelation method. The method involves a mixture of two aqueous phases, of which one is the polymer chitosan, a di-block co-polymer ethylene oxide or propylene oxide (PEO-PPO) and the other is a poly anion sodium tripolyphosphate. In this method, positively charged amino group of chitosan interacts with negative charged tripolyphosphate to form coacervates with a size in the range of nanometer. Coacervates are formed as a result of electrostatic interaction between two aqueous phases, whereas, ionic gelation involves the material undergoing transition from liquid to gel due to ionic interaction conditions at room temperature.

\section{Preparation of nanoparticles by polymerization of a monomer}

To attain the desired properties for a particular application, suitable polymer nanoparticles must be designed, which can be done during the polymerization of monomers. Processes for the production of PNPs through the polymerization of monomers are discussed below 


\section{Emulsion polymerization}

Emulsion polymerization is one of the fastest methods for nanoparticle preparation and is readily scalable. The method is classified into two categories, based on the use of an organic or aqueous continuous phase. ${ }^{[96]}$

The continuous organic phase methodology involves the dispersion of monomer into an emulsion or inverse microemulsion, or into a material in which the monomer is not soluble (nonsolvent). In the aqueous continuous phase the monomer is dissolved in a continuous phase that is usually an aqueous solution, and the surfactants or emulsifiers are not needed.

The polymerization can be initiated either by free radicals or by ion formation. The polymerization is initiated by the reaction of a monomer molecule with an initiator molecule.

Triggers for the initiation of the reaction can be ultraviolet (UV) light, hydroxyl ions, or highenergy radiation. The polymer chain starts to grow when these initiated monomer ions or monomer radicals react with other monomer molecules. Additional monomer is solubilized in surfactant micelles or emulsified in larger droplets. After completion of polymerization, the reaction mixture is filtered, neutralized, and purified by centrifugation to remove any residual monomer. An example for an anionic process is the preparation of "poly(alkylcyanoacrylate) nanoparticles" and an example for a free radical-initiated emulsion polymerization is the manutäcturing of polymethyl methacrylate nanoparticles [97]. The mechanism of an anionic polymerization is shown in Figure 7.

\section{Interfacial polymerization}

To achieve interfacial polymerization, monomers are polymerized at the interface between two immiscible phases.

Interfacial polymerization takes place in a medium consisting of an aqueous and an organic phase, which are homogenized, emulsified, or micro-fluidized by vigorously mechanical stirring. Al Kouhri Fallouh et al. ${ }^{[98]}$ introduced the formation of polyalkylcyanoacrylatnea nocapsules. In this process the monomer and the drug are dissolved in a mixture of oil and ethanol and then slowly added through a small tube or needle to an aqueous phase containing surfactants.

\section{Conclusion}

It emerges from this review that nanoparticles can easily enter brain capillaries before reaching the surface of the brain microvascular endothelial cells, under the condition that the surface of these colloids is modified in a proper way (i.e. by PEG or PS- 80). The prolonged blood circulation of these surface modified nanoparticles enhances exposure of the BBB, which favors interaction and penetration into brain endothelial cells.

There are now numerous preparation methods available for producing nanoparticles, and important technological advances have been achieved. Simple, safe, and reproduciblete chniques are now available to prepare drug-loaded nanospheres and nanocapsules. 
Production of NPs using the environmentally friendly processes like supercritical fluids is quite a promising area of research to develop the products that are free from the unwanted toxic residual solvents.

Depending on the physicochemical characteristics of a drug, it is now possible to choose the best method of preparation and the best polymer to achieve an efficient entrapment of the drug

In the past an increasing number of studies have been performed in order to better develop therapies against brain diseases. The major obstacle for good cerebral treatments is the inability of some drugs to cross the BBB, hence not being bioavailable for the interaction with brain target

This challenge has been approached using different strategies, consisting of direct drug delivery or a temporar yBBB opening. The drawback of these approaches is that they are too invasive for the patients. Thus, the Depending on the physicochemical characteristics of a drug, it is now possible to choose the best method of preparation and the best polymer to achieve an efficient entrapment of the drug approach represents one of the most interesting solutions.

Among colloids, polymeric $\mathrm{Np}$ have been rightly considered as promising carriers for CNS drug delivery, due to their potential both in encapsulating drugs, hence protecting them from excretion and metabolism within the body, andin delivering active agents across the BBB without inflicting any damage to the barrier.

Long circulation of drugs in the body is the key in successful drug delivery and drug targeting to the site of action

Many polymeric NPs have been developed for this purpose. Certainly, surface modification is useful in achieving these goals. From the polymer chemistry viewpoint, it is important to synthesize newer poly mers and copolymers to match the hydrophilic and hydrophobic properties. Production of NPs using the

A remarkable strategy is the use of different polymeric $\mathrm{Np}$ (such as the magnetic $\mathrm{Np}$ or the nanogels) or waxes (such as emulsifying wax or Brij 72), both to perform more specific and selective CNS drug delivery.

Specific ligands are commonly used in order to increase the specificity of drug delivery to the CNS, decreasing the loss of the drug over the peripheral blood circulation and improving the efficacy at the target site.

Different rationales drove the other approaches; in particular, the use of positively charged $\mathrm{Np}$ could be explained on the basis of their interaction with the negative charges of the BBB, while the use of surfactant (such as Polysorbate-80) and PEG is connected with the possible endocytic mechanism of BBB crossing and with the aim of prolonging the Np circulation half-life. 


\section{References}

1.W.M. Pardridge, Blood-brain barrier drug targeting: the future of brain drug development, Mol. Interv. 3 (2003) 90-105

2.Matthew Menken, MD; Theodore L. Munsat, MD; James F. Toole, MDThe Global Burden of Disease Studylmplications for Neurology Arch Neurol. 2000;57(3):418-420. doi:10.1001/archneur.57.3.418

3.Return on Investment: Mental Health Promotion and Mental Illness Prevention. Canadian Institute for Health Information. 2011.

4.World Health Organization. 2011. http://www.who.int/mental_health/media/en/382.pdf

5. Report by the WHO Secretariat on the Global Burden of Mental Disorders. WHO. 2011

6.4 World Alzheimer Report 2011. Alzheimer's Disease International. 2011. Available at: http://www.alz.co.uk/research/WorldAlzheimerReport2011.pdf

7. Indu Pal Kaur, Rohit Bhandari, Swati Bhandari, Vandita Kakkar .A Potential of solid lipid nanoparticles in brain targeting Journal of Controlled Release 127 (2008) 97-109

8.J. Kreuter, Nanoparticulate systems for brain delivery of drugs, Adv. Drug Deliv. Rev. 47 (2001) 65-81

9.Kenul Abbasova c, Rustam Berdiev c, Stefanie Wohlfart d, Nina Chepurnova c, Jörg Kreuter $\mathrm{d},{ }^{*}$ Drug delivery to the brain using surfactant-coated poly(lactide-co-glycolide) nanoparticles: Influence of the formulation parameters Research paper journal homepage: www.elsevier.com/locate/ejpb

10. M.INTAKHAB ALAM, Sarwar Beg,ABDUS Samad, Sanjula Bahooota,Kanchan Kohli, Javed Ali, AlkaAhuja,M. AKBAR. Strategy for effective brain drug delievery. Review European Jouranal of Pharmaceutical Science 40( 2010) 385-483.

11.E. Garcia-Garciaba , K. Andrieux a,* , S. Gil b , P. Couvreur a Colloidal carriers and bloodbrain barrier (BBB) translocation: A way to deliver drugs to the brain? Review International Journal of Pharmaceutics 298 (2005) 274-292

12. Chen D, Lee KH. 1993. Biodistribution of calcitonin encapsulated in liposomes in mice with particular reference to the central nervous system. Biochem Biophys Acta 1158:244-250

13. Kumaresh S. Soppimatha, Tejraj M. Aminabhavia , , Anandrao R. Kulkarnia, b Walter E. Rudzinski Biodegradable polymeric nanoparticles as drug delivery devices Review Journal of Controlled Release 70 (2001) 1-20.

14.Jean-Christophe Olivier Drug Transport to Brain with Targeted Nanoparticles NeuroRx: The Journal of the American Society for Experimental NeuroTherapeutics

15.Garcia-Garcia E, Andrieux K, Gil S,Couvreur P. Colloidal carriers and blood-brain barrier (BBB) translocation: a way to deliver drugs to the brain? Int J Pharm 2005 ; 298 : 274 -92 
16.D.J. Begley, Delivery of therapeutic agents to the central nervous system: the problems and the possibilities, Pharm. Ther. 104 (1) (2004) 29-45.

17. Neiman, Alcohol as a risk factor for brain damage: neurologic aspects,Alcohol Clin. Exp. Res. 22 (1998) S346-S351.

18. H. Wolburg, A. Lippoldt, Tight junctions of the blood-brain barrier: development, composition and regulation, Vasc. Pharmacol. 38 (2002) 323-337

19.U. Kniesel, H. Wolburg, Tight junctions of the blood-brain barrier, Cell. Mol. Neurobiol. 20 (2000) 57-76

20. W.M. Pardridge, P.L. Golden, Drug Delivery to the Central Nervous System, 1999.

21.N.J. Abbott, Astrocyte-endothelial interactions and blood-brain barrier permeability, J. Anat. 200 (2002) 629-63822.J.R. Gee, J.N. Keller, Astrocytes: regulation of brain homeostasis via apolipoprotein E, Int. J. Biochem. Cell Biol. 37 (2005) 1145-1150

22. W.M. Pardridge, P.L. Golden, Drug Delivery to the Central Nervous System, 1999.

23.J.R. Gee, J.N. Keller, Astrocytes: regulation of brain homeostasis via apolipoprotein E, Int. J. Biochem. Cell Biol. 37 (2005) 1145-1150.

24 .C.H. Lai, K.H. Kuo, The critical component to establish in vitro BBB model: pericyte, Brain Res. Brain Res. Rev. 50 (2005) 258-265.

25. R. Balabanov, P. Dore-Duffy, Role of the CNS microvascular pericyte in the blood-brain barrier, J. Neurosci. Res. 53 (1998) 637-644

26.B.T. Hawkins, T.P. Davis, The blood-brain barrier/neurovascular unit in health and disease, Pharmacol Rev. 57 (2005) 173-185

27.W.M. Pardridge, Brain Drug Targeting: The Future of Brain Drug Development, Cambridge University Press, Cambridge, UK, 2001.

28.E.M. Cornford, S. Hyman, Blood-brain barrier permeability to small and large molecules, Adv. Drug Deliv. Rev. 36 (1999) 145-163.

29.F.P. Bonina, L. Arenare, R. Ippolito, G. Boatto, G. Battaglia, V. Bruno, P. de Caprariis, Synthesis, pharmacokinetics and anticonvulsant activity of 7-chlorokynurenic acid prodrugs, Int. J. Pharm. 202 (2000) 79-88

30.W.M. Pardridge, CNS drug design based on principles of blood-brain barrier transport, J. Neurochem. 70 (1998) 1781-1792.

31. W.M. Pardridge, Vector-mediated drug delivery to the brain, Adv. Drug Deliv. Rev. 36 (1999) 299-321. 
32. W.M. Pardridge, P.L. Golden, Drug Delivery to the Central Nervous System, 1999.

33. D.W. Miller, A.V. Kabanov, Potential applications of polymers in the delivery of drugs to the central nervous system, Colloids Surf. B Biointerfaces 16 (1999) 321-330.

34. A.V. Kabanov, E.V. Batrakova, D.W. Miller, Pluronic block copolymers as modulators of drug efflux transporter activity in the blood-brain barrier, Adv. Drug Deliv. Rev. 55 (2003) 151164.

35. W. Sun, C. Xie, H. Wang, Y. Hu, Specific role of polysorbate 80 coating on the targeting of nanoparticles to the brain, Biomaterials 25 (2004) 3065-3071.

36. Abhilash M. Potential applications of Nanoparticles. Int J Pharm Bio Sci 1(1)2010.

37. Kayser.O, A. Lemke and N. Hernández-Trejo. (2005) The Impact of nanobiotechnology on the development of new drug delivery systems. Current Pharmaceutical Biotechnology 6(1),3

38.Y. Chen, G. Dalwadi, H.A.E. Benson, Drug delivery across the blood- brain barrier, Cur. Drug Deliv. 1 (2004) 361-376.

39. C.J. Van Oss, Phagocytosis as a surface phenomenon, Annu Rev. Microbiol. 32 (1978) 1939

40. H. Muller, K.M. Willis, Surface modification of i.v. injec able biodegradable nanoparticles with poloxamer polymers and poloxamine 908, Int. J. Pharm. 89 (1993) 25-31

41. Moghimi SM, Hunter AC, Murray JC. Long-circulating and target-specifi nanoparticles: theory to practice. Pharmacol Rev $2001 ; 53: 283-318$

42. L. Grislain, P Couvreur, V kanaerts, M. Roland, D. Deprez Decampeneerea,n d P.S peiserl,n t. J. Pharm.1 5,3 35 (1983). 98. J. Kreuter,P harm.A cta Helv.5 8,217( 1983).

43. Bazile DV, Ropert C, Huve P, et al. Body distribution of fully biodegradable [14C]-poly(lactic acid) nanoparticles coated with albumin after parenteraladministration to rats. Biomaterials1992 ; $13: 1093-102$

44. von Burkersroda F, Gref R, Gopferich A. E rosion of biodegradable block copolymers made of poly( D , L -lactid acid) and poly(ethylene glycol). Biomaterials 1997 ; 18 : 1599 -607

45. Stolnik S, Dunn SE, Garnett MC, et al. Surface modifi cation of poly(lactide-co-glycolide) nanospheres by biodegradable poly(lactide)-poly(ethylene glycol) copolymers. Pharm Res $1994 ; 11: 1800-8$

46. Verrecchia T, Spenlehauer G, Bazile DV, et al. Non-stealth (poly(lactic acid/albumin)) and stealth (poly(lactic acid-polyethylene glycol)) nanoparticles as injectable drug carriers. J Control Rel $1995 ; 36: 49-61$ 
47. Le Ray AM, Vert M, Gautier JC, Beno î t JP. Fate of [14C]poly(-lactide-co-glycolide) nanoparticles after intravenous and oral administration to mice. Int J Pharm $1994 ; 106: 201-11$

48. S.M.Moghimi, C.Hunter, J.C.Murray, Long-circulating and target-specific nanoparticles: theory to Practice, Pharmacol Rev. 53 (2001) 283-318

49. D. Chen, T. Yang, W. Liang, Q. Zhang, In vitro and in vivo study of two types of long circulating solid lipid nanoparticles containing Paclitaxel, Chem. Pharm. Bull. 49 (2001) 14441447

50.M.O. Oyewumi, R.A. Yokel, M. Jay, T. Coakley, R.J. Mumper, Comparison of cell uptake, biodistribution and tumor retention of folate coated and PEG coated gadolinium nanoparticles in tumor bearing mice, J. Control. Release 95 (2004) 613-626.

51. Gref R, Domb A, Quellec $P$, et al. The controlled intravenous delivery of drugs using PEGcoated sterically stabilized nanospheres. Adv Drug Del Rev 1995 ; 16 : 215 -33

52.R. Cavalli, O. Caputo, M.R. Gasco, Preparation and characterization of solid lipid nanospheres containing paclitaxel, Eur. J. Pharm. Biopharm. 10 (2000) 305-330

53. Koziara JM, Lockman PR, Allen DD, Mumper RJ. Paclitaxel nanoparticles for the potential treatment of brain tumors. J Control Rel $2004 ; 30: 259-69$

54. U. Schroeder, B. A. Sabel, and H. Schroeder, Life Sci. 66,495 (2000).

55.G. Borchardt, L.A. Kenneth, S. Fenlin, J. Kreuter, Uptake of surfactant-coated poly(methyl methacrylate)-nanoparti cles by bovine brain microvessel endothelial cell mono layers, Int. J. Pharmacol. 110 (1994) 29-35.

56.A.E. Gulyaev, S.E. Gelperina, I.N. Skidan, A.S. Antropov, G.Y. Kivman, J. Kreuter, Significant transport of doxorubi cin into the brain with polysorbate 80-coated nanoparticles Pharm. Res. 16 (1999) 1564-1569

57.R.N. Alyautdin, V.E. Petrov, K. Langer, A. Berthold, D.A. Kharkevich, J. Kreuter, Delivery of lopermide across the blood-brain barrier with polysorbate 80-coated poly 25 328.butylcyanoacrylate nanoparticles, Pharm. Res. 14 (1997)

58. R.N. Alyautdin, E.B. Tezikov, P. Ramga, D.A. Kharkevich D.J. Begley, J. Kreuter, Significant entry of tubocurarin into the brain of rats by adsorption to polysorbate 80-coated glypolybutylcyanoacrylate nanoparticles: in situ brain perfu- sion study, J. Microencapsulation 15 (1998) 67-74

59. J. Kreuter, Nanoparticulate system for brain delivery of drugs, Adv. Drug Deliv. Rev. 47 (2001) 65-81. 
60. T.M. Goppert, R.H. Müller, Polysorbate-stabilized solid lipid nanoparticles as colloidal carriers for intravenous targeting of drugs to the brain: comparison of plasma protein adsorption patterns, J. Drug Target. 13 (3) (2005) 179-187.

61. B. Dehouck,L . Fenart,M .-P Dehouck,A . Pierce,G . Torpier,a nd R. CecchelliJ, . CellB iol. 138,8 77 (1997)

62. J.M. Harris, R.B. Chess, Effect of pegylation on pharmaceuticals, Nat.Rev. Drug Discov. 2 (2003) 214-221

63. Yamazaki T, Sato Y, Hanai M, et al. Non-ionic detergent tween 80 modulates VP-16 resistance in classical multidrug resistant K562 cells viaenhancement of VP-16 infl ux. Cancer Lett $2000 ; 149: 153-61$

64. Anderberg EK, Nystr ö m C, Artursson P. Epithelial transport of drugs in cell culture.

VII: effects of pharmaceutical surfactant excipients and bile acids on transepithelial permeability in monolayers of human intestinal epithelial (Caco-2) cells. J Pharm Sci $1992 ; 81: 879$-87

65. Vyas SP, Singh A, Sihorkar V. Ligand-receptor mediated drug delivery: an emerging paradigm in cellular drug targeting. Crit Rev Ther Drug Carr Syst 2001; 189 : 1 -76

66. Newton HB. Advances in strategies to improve drug delivery to brain tumors. Exp Rev Neurother $2006 ; 6: 1495-509$

67. M.L. Hans, A.M. Lowman, Biodegradable nanoparticles for drug delivery and targeting, Curr. Opin. Solid State Mater. Sci. 6 (2002) 319-327. Journal of Controlled Release journal homepage: www.elsevier.com/locate/jconrel Review

68.PLGA-based nanoparticles: An overview of biomedical applications Fabienne Danhier a, Eduardo Ansorena a, Joana M. Silva a,b, Régis Coco, Aude Le Breton, Véronique Préat

69. Catarina Pinto Reis, Ronald J. Neufeld, Antonio J. Ribeiro, Francisco Veiga. Nanoencapsulation I. Methods for preparation of drug-loaded polymeric nanoparticles Nanomedicine: Nanotechnology, Biology, and Medicine 2 (2006) 8- 21.

70. Fessi H, Puisieux F, Devissaguet J-P, Ammoury N, Benita S. Nanocapsule formation by interfacial deposition following solvent displacement. Int J Pharm 1989;55:R1- R4.

71. Ganachaud F, Katz JL. Nanoparticles and nanocapsules created using the ouzo effect: Spontaneous emulsification as an alternative to ultrasonic and high-shear devices. Chem Phys Chem 2005;6: 
72.Quintanar-Guerrero D, Alle'mann E, Fessi H, Doelker E. Preparation techniques and mechanism of formation of biodegradable nanoparticles from preformed polymers. Drug Dev Ind Pharm 1998;24: 1113-28.

73. Wehrle $\mathrm{P}$, Magenheim $\mathrm{B}$, Benita $\mathrm{S}$. Influence of process parameters on the PLA nanoparticle size distribution, evaluated by means of factorial design. Eur $\mathrm{J}$ Pharm Biopharm 1995;41:19- 26.

74.Ne'mati F, Dubernet C, Fessi H, Verdie're AC, Poupon MF, Puisieux F, et al. Reversion of multidrug resistance using nanoparticles in vitro: influence of the nature of the polymer. Int $\mathrm{J}$ Pharm 1996;138: 237- 46

75.Barichello JM, Morishita M, Takayama K, Nagai T. Encapsulation of hydrophilic and lipophilic drugs in PLGA nanoparticles by the nanoprecipitation method. Drug Dev Ind Pharm 1999;25:471- 6., PCL

76. Molpeceres J, Guzman M, Aberturas MR, Chacon M, Berges L. Application of central composite designs to the preparation of polycaprolactone nanoparticles by solvent displacement. J Pharm Sci 1996;85:206 - 13.

77.Irache JM, Huici M, Konecny M, Espuelas S, Campanero MA, Arbos P. Bioadhesive properties of gantrez nanoparticles. Molecules 2005;10:126 - 45.

78.Arbo's P, Wirth M, Arangoa MA, Gabor F, Irache JM. GantrezRAN as a new polymer for the preparation of ligand-nanoparticle conjugates. J Control Release 2002;83:321- 30

79.Seijo B, Fattal E, Roblot-Treupel L, Couvreur P. Design of nanoparticles of less than $50 \mathrm{~nm}$ diameter: preparation, characterization and drug loading. Int J Pharm 1990;62:1 - 7.

80.Ammoury N, Fessi H, Devissaguet J-P, Dubrasquet M, Benita S. Jejunal absorption, pharmacological activity, and pharmacokinetic evaluation of indomethacin-loaded poly(d,Ilactide) and poly(isobutylcyanoacrylate) nanocapsules in rats. Pharm Res 1991;8:101- 5.

81.T. Niwa, H. Takeuchi, T. Hino, N. Kunou, Y. Kawashima Preparations of biodegradable nanospheres of water-soluble and insoluble drugs with D, L-lactide / glycolide copolymer by a novel spontaneous emulsification solvent diffusion method and the drug release behavior, J. Control. Rel. 25 (1993) 89-98.

82. P. Wehrle, B. Magenheim, S. Benita, The Influence of process parameters on the PLA nanoparticle size distribution evaluated by means of factorial design, J. Pharm. Biopharm 41 (1995) 19-26

83.Niwa T, Takeuchi H, Hino T, Kunou N, Kawashima Y. Preparation of biodegradable nanoparticles of water-soluble and insoluble drugs with $\mathrm{D}$, Llactide/ glycolide copolymer by a novel spontaneous emulsification solvent diffusion method, and the drug release behavior. J. Control.Release 1993; 25: 89-98 
84.Catarina Pinto Reis, Ronald J. Neufeld, Antonio J. Ribeiro, Francisco Veiga. Nanoencapsulation I. Methods for preparation of drug-loaded polymeric nanoparticles Nanomedicine: Nanotechnology, Biology, and Medicine 2 (2006) 8-21.

85.Quintanar-Guerrero D, Alle'mann E, Fessi H, Doelker E. Preparation techniques and mechanism of formation of biodegradable nanoparticles from preformed polymers. Drug Dev Ind Pharm 1998;24: 1113-28.

86. Jung T, Kamm W, Breitenbach A, Kaiserling E, Xiao JX, Kissel T. Biodegradable nanoparticles for oral delivery of peptides: is there a role for polymers to affect mucosal uptake? Eur J Pharm Biopharm 2000;50:147-60

87.Lambert G, Fattal E, Couvreur P. Nanoparticulate system for the delivery of antisense oligonucleotides. Adv Drug Deliv Rev 2001; 47:99 - 112.

88.York P. Strategies for particle design using supercritical fluid technologies. Pharm Sci Technol Today 1999; 2:430-40.

89. K. Mishima, K. Matsuyama, D. Tanabe, S. Yamauchi, [55] F. Lescure, C. Seguin, P. Breton, P. Bourrinet, D. Roy, P. Microencapsulation of proteins by rapid expansion of super- Couvreur, Preparation and charecterization of novel polycritical solution with a nonsolvent, AIChE J. 46 (2000) (methylidene malonoate 2.1.2.)-made nanoparticles, Pharm. 857-865. Res. 11 (1994) 1270-1277.

90.J.W. Tom, P.G. Debenedetti, Formation of bioerodiable polymeric microspheres and microparticles by rapid expansionof supercritical solution, Biotechnol. Prog. 7 (1991) 403-411. nanoparticles, Biomaterials 19 (1998) 271-281.

91.J.W. Tom, P.G. Debenedetti, R. Jerome, Preparation of [poly(L-lactic acid) and composite poly(L-lactic acid)-pyrene mby rapid expansion of supercritical solution, J. Supercrit. Fluids 7 (1994) 9-29.

92.T.W. Randolph, A.D. Randolph, M. Mebes, S. Yeung, Sub- micron-sized biodegradable particles of poly(L-lactic acid) via the gas antisolvent spray precipitation process, Biotech- nol. Prog. 9 (1993) 429-435.

93.Calvo P, Remunan-Lopez C, Vila-Jato JL, Alonso MJ. Novel hydrophilic chitosanpolyethylene oxide nanoprticles as protein carriers. J. Appl. Polymer Sci. 1997; 63: 125- 132.

94. Calvo P, Remunan-Lopez C, Vila-Jato JL, Alonso MJ. Chitosan and chitosan/ethylene oxide-propylene oxide block copolymer nanoparticles as novel carriers for proteins and vaccines. Pharm Res. 1997; 14: 1431-1436.

95. Amir Dustgania, Ebrahim Vasheghani Farahania, Mohammad Imanib. Preparation of Chitosan Nanoparticles Loaded by Dexamethasone Sodium Phosphate. Iranian J Pharma Sci 2008, 4(2): 111-114. 
96. Kreuter J. Large-scale production problems and manufacturing of nanoparticles. In: Tyle P, editor. Specialized drug delivery system. New York7 Marcel Dekker; 1990. p. 257- 66.

97. J. Kreuter, "Encyclopedia of Pharmacy Technology," 165 (1994). p. 257. Marcel Dekker, New York, 1990. 67. J.Kreuter, in "SpecializedD rug DeliveryS ystems("P Tyle,E d.), 68. G. Birrenbacha nd P P SpeiserJ, . Pharm. Sci. 65,1763 (1976).

98.N. Al Khouri Fallouh, L. Roblot:Treubel, H. Fessi, J. P. Devis- (1999). saguet, and E Puisieux, Int. I. Pharm. 28, 125 (1986).

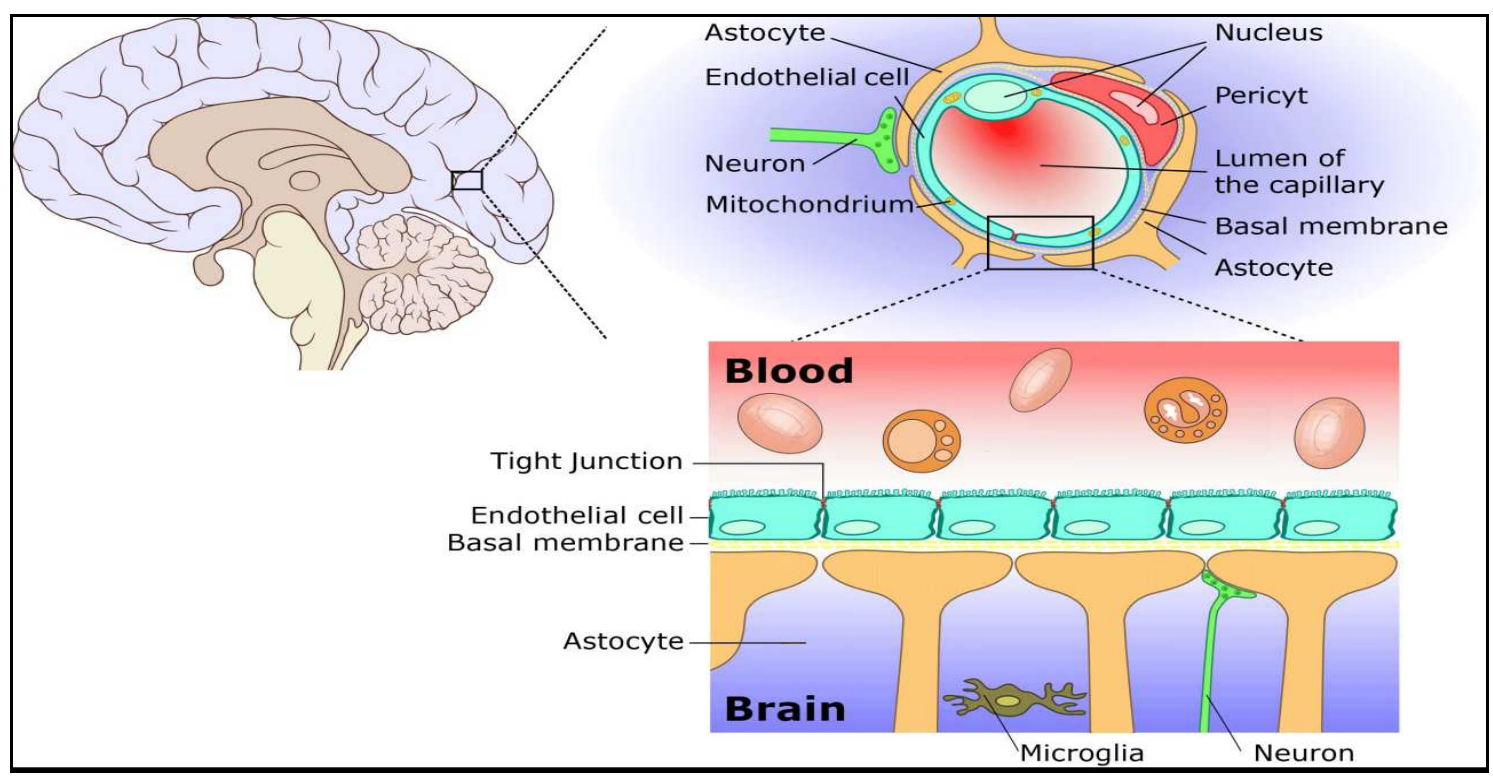

Figure 1: The blood-brain barrier; above, cross section through the brain; center, schematic representation of the BBB; 
Organic solution:

Polymer + Drug in water non-miscible solvent

II Aqueous solution:

Subilizer in watler

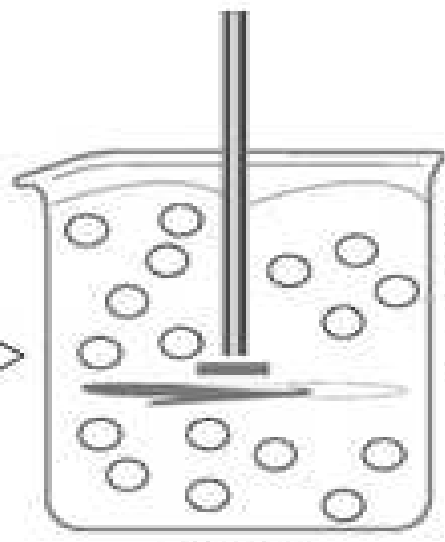

Step I

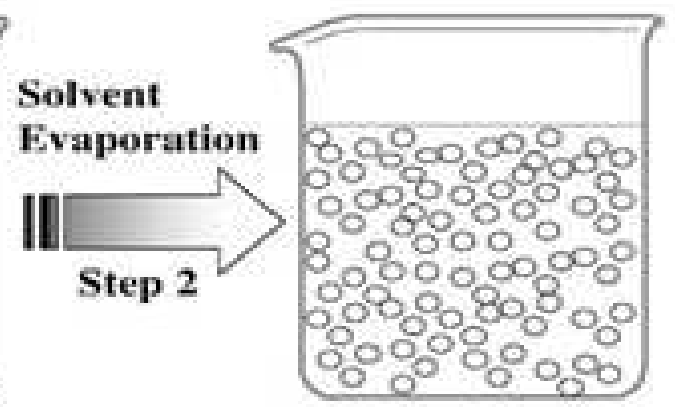

Figure 2: Schematic representation of the solvent-evaporation technique [Ref: Catarina Pinto Reis et al 69].

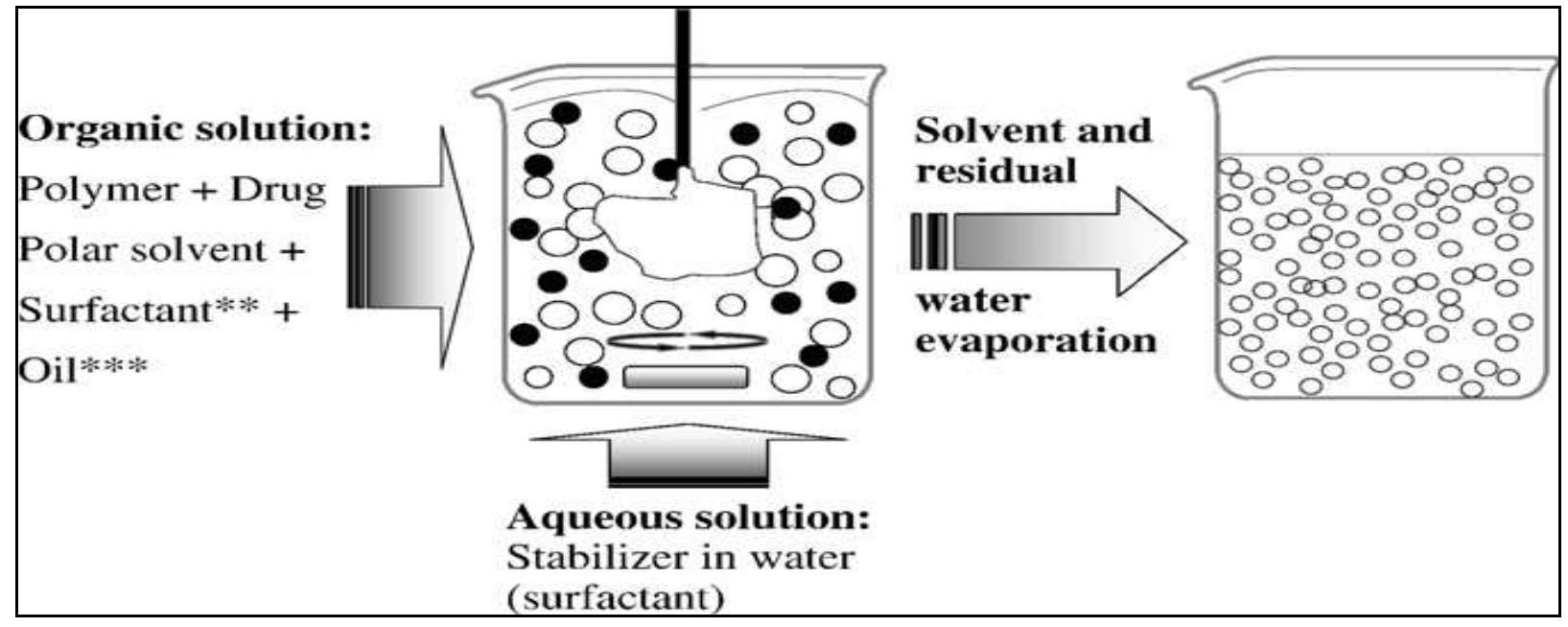

Figure 3. Schematic representation of the solvent displacement technique 


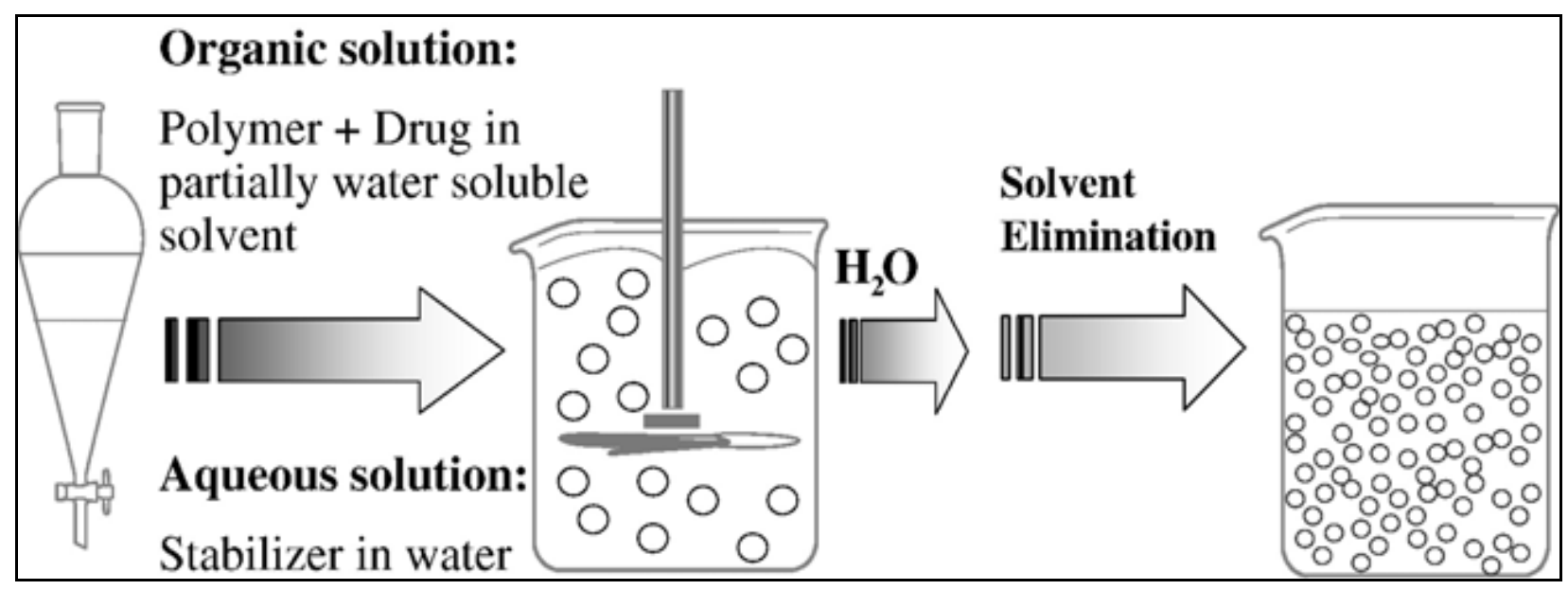

Figure 4: Schematic illustration of the ESD technique

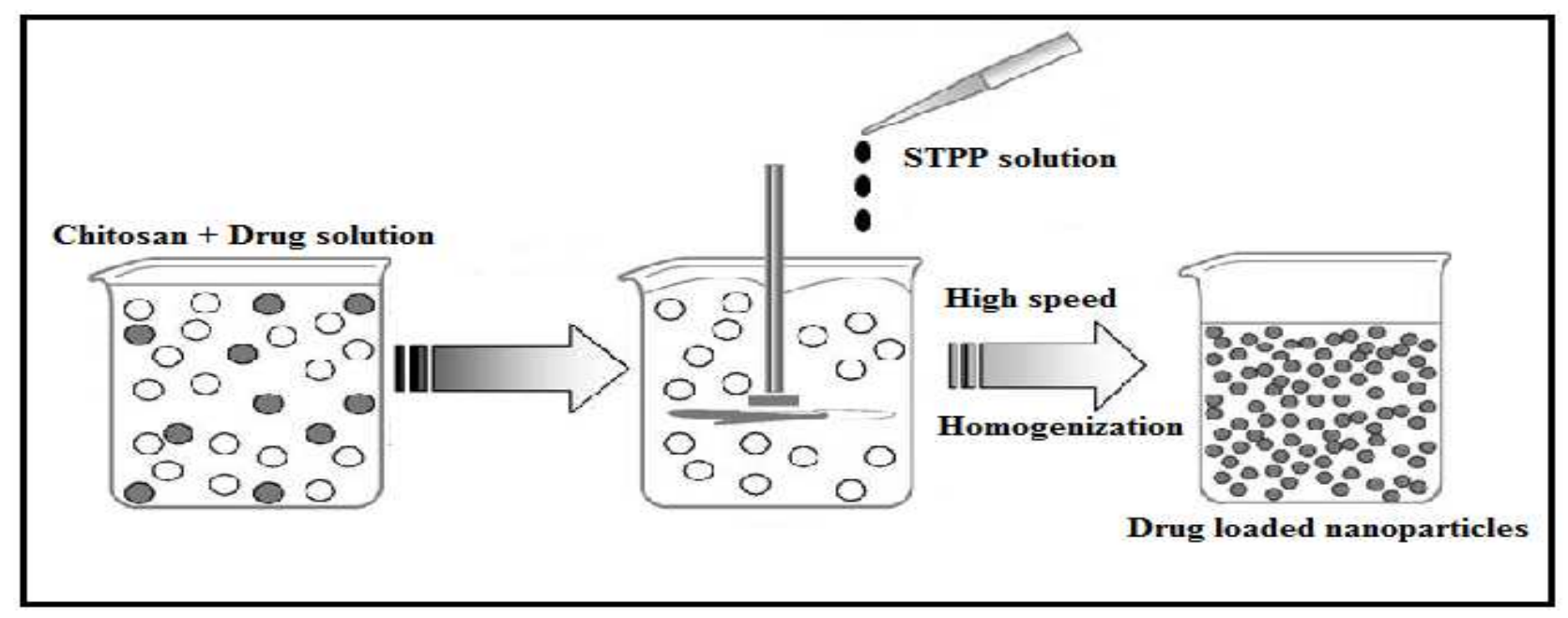

Figure 5: Schematic of the salting-out technique 


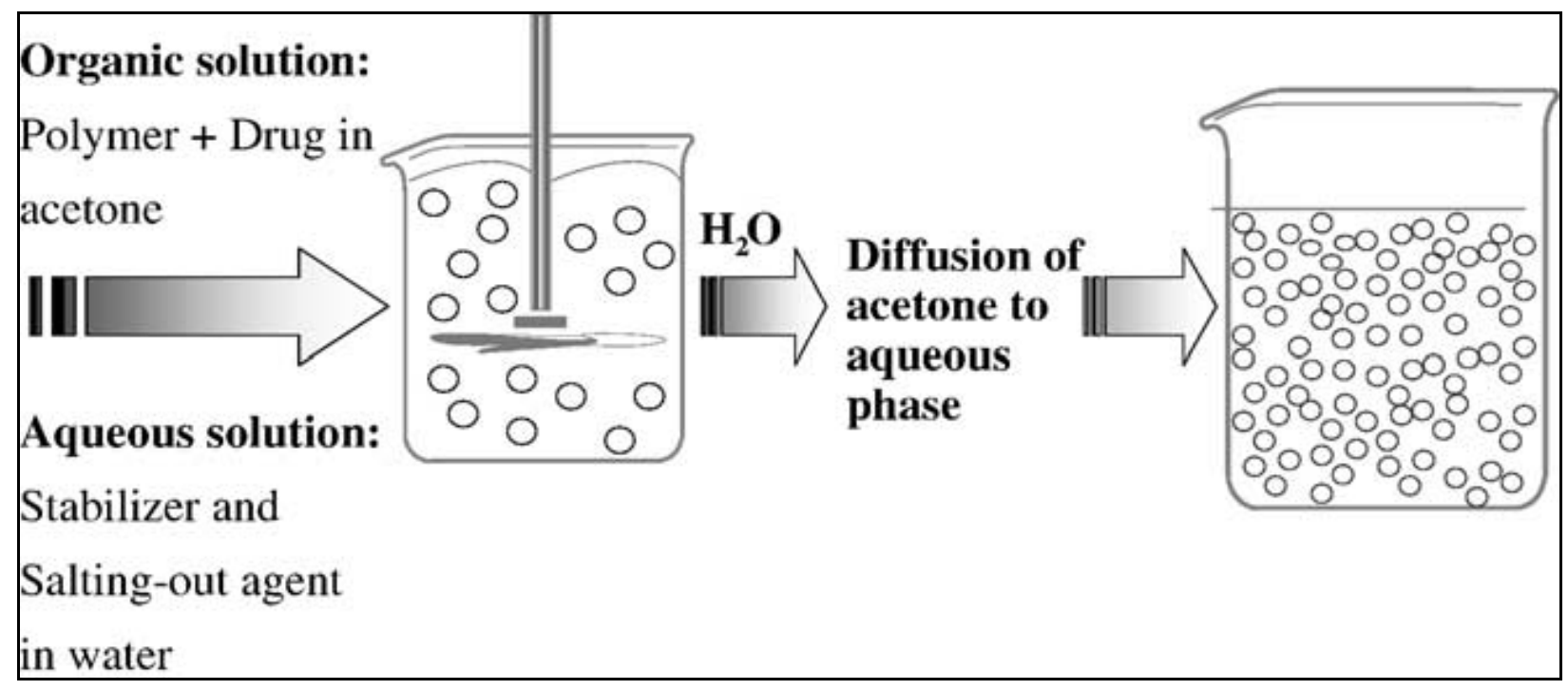

Figure 6: Schematic representation of ionic gelation method

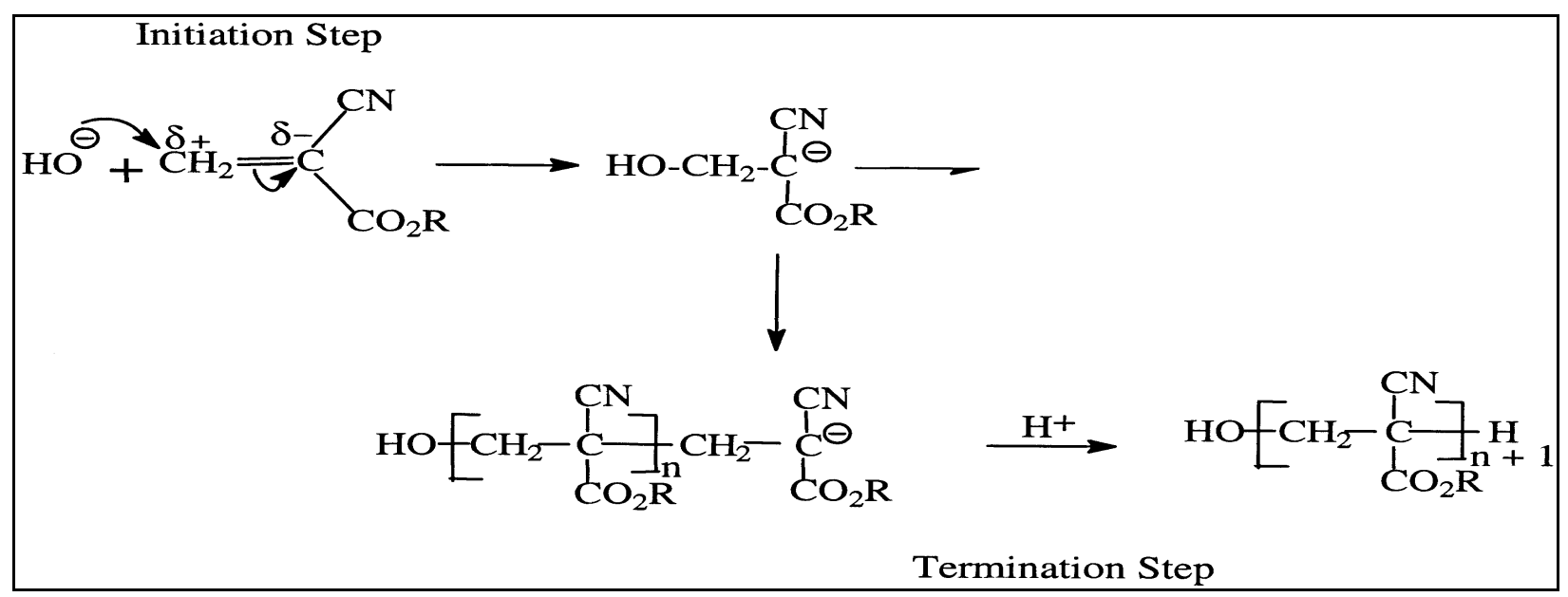

Figure 7: The mechanism of an anionic polymerization 